\title{
Energetischer Aufwand der Bereitstellung von Primärkupfer für Deutschland
}

\author{
Nadine Rötzer ${ }^{1}$ iD \\ Eingegangen: 1. April 2021 / Überarbeitet: 22. September 2021 / Angenommen: 27. September 2021 / Online publiziert: 2. November 2021 \\ (c) Der/die Autor(en) 2021
}

\section{Zusammenfassung}

Die geologisch verfügbare Menge an Metallen wie Kupfer wird kontrovers diskutiert. Die Rohstoffpolitik sollte sich besser am eigentlich einschränkenden Faktor, der erforderlichen Energie zur Rohstoffbereitstellung, statt an dieser spekulativen Diskussion orientieren. Dazu ist ein Verständnis des energetischen Aufwands und seiner Einflussfaktoren unerlässlich. In der vorliegenden Arbeit wird ein generisches Modell der Kupfergewinnung vorgestellt und der kumulierte Energieaufwand (KEA) sowie das Treibhausgaspotential (GWP) der Primärkupferbereitstellung für Deutschland ermittelt. Der mittels des Modells berechnete KEA beträgt $40 \mathrm{MJ} / \mathrm{kg} \mathrm{Cu}$, das $\mathrm{GWP} 3,3 \mathrm{~kg} \mathrm{CO}$ - $\mathrm{Äq} / \mathrm{kg} \mathrm{Cu}$. Eine detaillierte Betrachtung zeigt, dass KEA und GWP des importierten Kupfers abhängig von der Bezugsquelle deutlich variieren. Die Bezugsquelle und die mit ihr einhergehenden geologischen und technischen Parameter stellen somit wichtige Einflussfaktoren hinsichtlich des energetischen Aufwands und der Emission von Treibhausgasen dar. Neben den Bezugsquellen spielt auch die Art des importierten Guts, d.h. Kupferkonzentrat oder Kupferkathode, eine Rolle. Der Transportaufwand ist beim Import von Kupferkonzentrat aufgrund dessen geringen Metallgehalts deutlich höher. Unter gleichen technischen Voraussetzungen ist es daher sinnvoll, dass die metallurgische Behandlung möglichst in der Nähe der Minen stattfindet. Dies gilt es jedoch im Einzelfall und unter Berücksichtigung weiterer z. B. rohstoffpolitischer Aspekte zu prüfen.

Schlüsselwörter Energieaufwand · Treibhausgasemissionen · Rohstoffverfügbarkeit · Deutschland · Kupfer

\section{Energy demand of the supply of primary copper for Germany}

\begin{abstract}
The geologically available amount of metals such as copper is discussed controversially. Raw materials policy should be guided by the actual limiting factor, the energy required to provide raw materials, rather than by this speculative discussion. Therefore, an understanding of the energetic costs and its influencing factors is essential. In this paper, a generic model of copper production is presented. With the model the cumulative energy demand (CED) and the greenhouse gas potential (GWP) of the supply of primary copper for Germany are determined. The CED calculated is $40 \mathrm{MJ} / \mathrm{kg} \mathrm{Cu}$, the GWP $3.3 \mathrm{~kg} \mathrm{CO} 2-\mathrm{eq} / \mathrm{kg} \mathrm{Cu}$. A detailed analysis shows that the CED and GWP of imported copper vary significantly depending on the source of supply. Therefore, the source of supply and the geological and technical parameters associated with it represent important influencing factors regarding the energetic effort and the emission of Greenhouse gases. The type of imported goods, i.e. copper concentrate or cathode, also plays a role. The transport effort is higher when importing copper concentrate due to its low metal content. If technical conditions are the same, metallurgical treatment should therefore take place near the mines. However, this must be examined on a case-by-case basis, taking into account further aspects such as raw materials policy.
\end{abstract}

Keywords Energy demand · Global warming potential $\cdot$ Resource availability $\cdot$ Germany $\cdot$ Copper

Nadine Rötzer

nadine.roetzer@hs-pforzheim.de
Institute for Industrial Ecology, Hochschule Pforzheim, Pforzheim, Deutschland 


\section{Einleitung}

Kupfer ist in der menschlichen Zivilisation kaum mehr wegzudenken. Der Kupferbedarf wird durch die wachsende Weltbevölkerung, das sich angleichende Wohlstandsniveau und neue Technologien wie die erneuerbare Energieerzeugung weiterhin steigen (Kleijn et al. 2011; MarscheiderWeidemann et al. 2016; Hilgers und Becker 2020). Jedoch ist die in unserer Erdkruste verfügbare Menge begrenzt, da Kupfer wie alle Metalle zu den nicht-erneuerbaren Rohstoffen gehört.

Die Diskussion um die Verfügbarkeit von Rohstoffen wurde insbesondere durch den vom Club of Rome veröffentlichten Bericht „Die Grenzen des Wachstums“ populär (Meadows et al. 1972). Dieser wies zu Beginn der 1970erJahre darauf hin, dass wir in naher Zukunft an die Grenzen der geologischen Rohstoffverfügbarkeit stoßen könnten. Grundlage für die damals durchgeführten Berechnungen waren jedoch die sogenannten Reserven der Rohstoffe, was vielfach $\mathrm{zu}$ falschen Interpretationen führte. Die Reserven sind nämlich die Teilmenge der gesamten in unserer Erdkruste befindlichen Menge, die bekannt und unter den derzeitigen Bedingungen wirtschaftlich gewinnbar ist. Sie stellen somit eine Momentaufnahme dar, auf deren Basis sich keine Aussagen über die zukünftige Rohstoffverfügbarkeit treffen lassen. Geeigneter scheinen hier die von Geologen sogenannten Ressourcen zu sein, welche jene Menge ist, die vermutet wird bzw. noch nicht wirtschaftlich abbaubar ist, deren Qualität aber für eine zukünftige Gewinnung ausreicht. Doch auch sie sind eine Momentaufnahme, da die Erde bei Weitem noch nicht vollständig exploriert ist. Dies zeigt sich auch an der großen Bandbreite aktueller Angaben zu den globalen Kupferressourcen. Die Schätzungen reichen von knapp 2 Mrd. t (Gerst 2008; Mudd et al. 2013; Northey et al. 2014) über 5,6 Mrd. t (Johnson et al. 2014) bis zu 89Mrd. t (Kesler und Wilkinson 2008).

Ein weiterer Aspekt, der immer wieder in der Diskussion um die Rohstoffverfügbarkeit angeführt wird, sind die sinkenden Erzgehalte abgebauter Lagerstätten (z.B. Vieira et al. 2012). Dass die abgebauten Erzgehalte sinken, zeigen zahlreiche Arbeiten wie Mudd (2009) oder Calvo et al. (2016). Jedoch sind sie nicht auf eine Erschöpfung der natürlichen Ressourcen zurückzuführen. Dies kann am Beispiel Kupfer verdeutlicht werden: Der steigende Bedarf führte im Zeitverlauf zum Abbau anderer Lagerstättentypen, die sich durch ihre enorme Größe und oberflächennahe Lage und einen geringen Erzgehalt auszeichnen. Dieser Trend war mit einer Weiterentwicklung der eingesetzten Abbaumethoden und Technologien verbunden. Sinkende Erzgehalte sind daher vielmehr ein Indikator für Skaleneffekte sowie den technologischen Fortschritt und nicht für die Erschöpfung der natürlichen Ressourcen (Rötzer und Schmidt 2018).
Mengenbasierte Indikatoren wie die Ressourcen oder Erzgehalte sind deshalb wenig aussagekräftig. Zudem werden Metalle nicht verbraucht, sondern gebraucht. Nach ihrer Nutzung stehen sie theoretisch wieder zur Verfügung. Jedoch geht ihre Nutzung mit einer Dissipation einher, deren Umkehrung mit einem hohen energetischen Aufwand verbunden ist. Technologiemetalle werden häufig in kleinsten Mengen in unseren Produkten verbaut. Diese geringen Konzentrationen der Metalle spiegeln sich im energetischen Aufwand der Rückgewinnung wider (Schäfer und Schmidt 2020).

Auch die Primärgewinnung von Metallen ist mit einem energetischen Aufwand verbunden, der maßgeblich durch den Erzgehalt beeinflusst wird. Der Zusammenhang zwischen energetischem Aufwand und Erzgehalt lässt sich mit einer 1/x Kurve beschreiben (Valero 2008, S. 160; Chapman und Roberts 1983; Northey et al. 2013).

Entscheidend ist also, welcher Aufwand betrieben werden muss, um Metalle zu gewinnen. Um diesen Aufwand zu erfassen, eignet sich die Energie. Sie stellt die begrenzende Ressource da, da sie notwendig ist, um die Konzentration von Stoffen in einem definierten System zu erhöhen. Je geringer die Konzentration, desto höher der notwendige Energieeinsatz. Wäre unendlich Energie verfügbar, so könnte man z.B. auch Metalle aus der durchschnittlichen Erdkruste gewinnen - es gäbe kein Rohstoffproblem. Mit dem Einsatz von Energie sind wiederum Kosten und Umweltwirkungen verbunden. Die Energie stellt auch hier ein verbindendes Element dar.

Von besonderem Interesse hinsichtlich der Umweltwirkungen sind die Treibhausgasemissionen, deren Reduktion Schwerpunkt zahlreicher politischer Aktivitäten ist. Diese werden aufgrund ihrer hohen Relevanz zusätzlich berechnet.

Auch für Deutschland als Industrie- und Exportstandort ist eine sichere und nachhaltige Rohstoffversorgung von hoher Bedeutung, weshalb die Bundesregierung 2010 und 2020 Rohstoffstrategien beschlossen hat (BMWi 2019). Darin wird u. a. eine ökonomisch, ökologisch und sozial verantwortungsvolle Rohstoffgewinnung gefordert. Im Gegensatz zu anderen Ländern wie Polen oder Chile ist Deutschland jedoch seit der Schließung des Kupferbergbaus im Mansfelder Revier im Jahr 1990 von Kupferimporten vollständig abhängig. Nur ein Teil des Kupferbedarfs kann bereits durch Recycling gedeckt werden.

Im Jahr 2010 wurden in Deutschland rund 1,3 Mio. t $\mathrm{Cu}$ nachgefragt (Dorner 2013). Durch den Import von raffiniertem Kupfer wurden 0,7 Mio. $\mathrm{t} \mathrm{Cu}$ bereitgestellt. $\mathrm{Zu}$ sätzlich wurden 1,1 Mio. t Kupferkonzentrat importiert, dies entspricht bei einem angenommenen Kupfergehalt von durchschnittlich ca. $30 \%$ rund 0,3 Mio. t $\mathrm{Cu}$. Des Weiteren wurde eine geringe Menge unraffiniertes Kupfer importiert (63 Tsd. t Cu). Die verbleibenden rund 0,2 Mio. t $\mathrm{Cu}$ wur- 


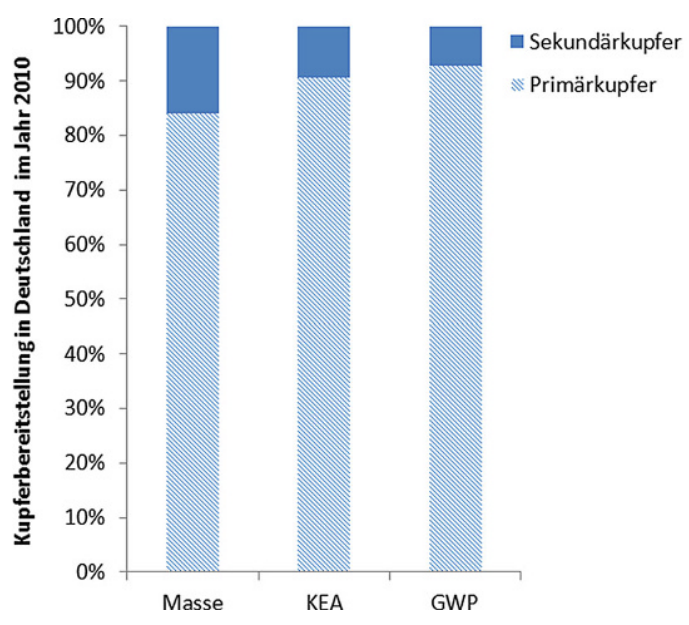

Abb. 1 Beitrag des Primär- und Sekundärkupfers zum KEA und GWP der Kupferbereitstellung für Deutschland im Jahr 2010. (Quellen: GWP und KEA Sekundärkupfer: Schäfer und Schmidt 2020; KEA und GWP Primärkupfer: ICA 2017; Menge Primärkupfer und Sekundärkupfer (Schätzung): Dorner 2013)

den durch Recycling bereitgestellt. Seiter ist der Anteil an Sekundärkupfer jedoch kontinuierlich gestiegen und betrug im Jahr 2019 bereits 44\% (BGR 2020).

Bewertet man für das Jahr 2010 die jeweiligen Mengen an Primär- und Sekundärkupfer mit in der Literatur verfügbaren durchschnittlichen Angaben zum kumulierten Energieaufwand (KEA) bzw. zum Treibhausgaspotential (GWP) (Schäfer und Schmidt 2020; ICA 2017), dann ergibt sich für die Kupferbereitstellung ein KEA von ca. 55 TJ sowie ein GWP von rund 4,7Mio.t $\mathrm{CO}_{2}$ - $\mathrm{Äq}$. Das Primärkupfer trägt jeweils mit mindestens $90 \%$ zu den beiden Kategorien bei, der mengenmäßige Anteil des Primärkupfers liegt bei $84 \%$ (siehe Abb. 1). Der leicht höhere Beitrag zum KEA und GWP im Vergleich zum Massenanteil ist darauf zurückzuführen, dass das aus dem Recycling bereitgestellte Kupfer pro Masseneinheit mit einem geringeren KEA bzw. GWP verbunden ist. Zur Senkung des Aufwands (KEA und GWP) der Kupferbereitstellung und damit zur Leistung ei- nes Beitrags zum Klimaschutz bietet die Gewinnung von Kupfer aus primären Quellen unter den aktuellen Bedingungen einen deutlich größeren Ansatzpunkt.

Zur Identifikation von technischen Optimierungspotenzialen in der Primärgewinnung und zur Erstellung von Prognosen ist es unerlässlich, die Einflussfaktoren zu verstehen. Dies ist durch die Verwendung der verfügbaren globalen Durchschnittswerte nicht möglich. Deutschland importiert Kupfer bzw. Kupferkonzentrat aus unterschiedlichen Regionen (siehe Abb. 2). In jeder Region liegt Kupfer unter anderen geologischen Bedingungen vor, welche einen Einfluss auf die eingesetzten Verfahren sowie den damit verbundenen Aufwand haben. Zudem spielt die Stromerzeugung in den einzelnen Ländern eine wichtige Rolle. Um Maßnahmen zur Reduktion des Aufwands abzuleiten, bedarf es einer Bewertung, welche die spezifischen Gegebenheiten einzelner Bezugsquellen bzw. Länder berücksichtigt und ihren Einfluss auf den Aufwand aufzeigt. Dazu wird in dieser Arbeit ein modellbasierter Ansatz vorgestellt.

\section{Methode}

\subsection{Modell zur Ermittlung des Aufwands}

Der KEA wird als zentraler Aufwandsindikator verwendet. Dabei wird sowohl der nicht-erneuerbare als auch der erneuerbare Anteil berücksichtigt. Der nicht-erneuerbare KEA ist insbesondere für die Emission von Treibhausgasen relevant. Da jedoch die Energie insgesamt ein Mangelfaktor ist, wird der erneuerbare KEA ebenfalls in die Betrachtung einbezogen. Zur Ermittlung des KEA sowie des GWP wird ein prozessbasiertes Modell für die Kupfergewinnung erstellt. Ähnliche Ansätze wurden bereits in den 1970er- und 1980er-Jahren von Chapman (1973) und Gaines (1980) angewandt. Auch aktuelle Arbeiten wie Norgate und Rankin (2000), Norgate und Jahanshahi (2010) oder Marsden (2008) nutzen prozessbasierte Modelle zur Ermittlung des
Abb. 2 Bezugsquellen von Kupferkonzentrat und Kupferkathoden. (Dorner 2013 nach BGR 2012)
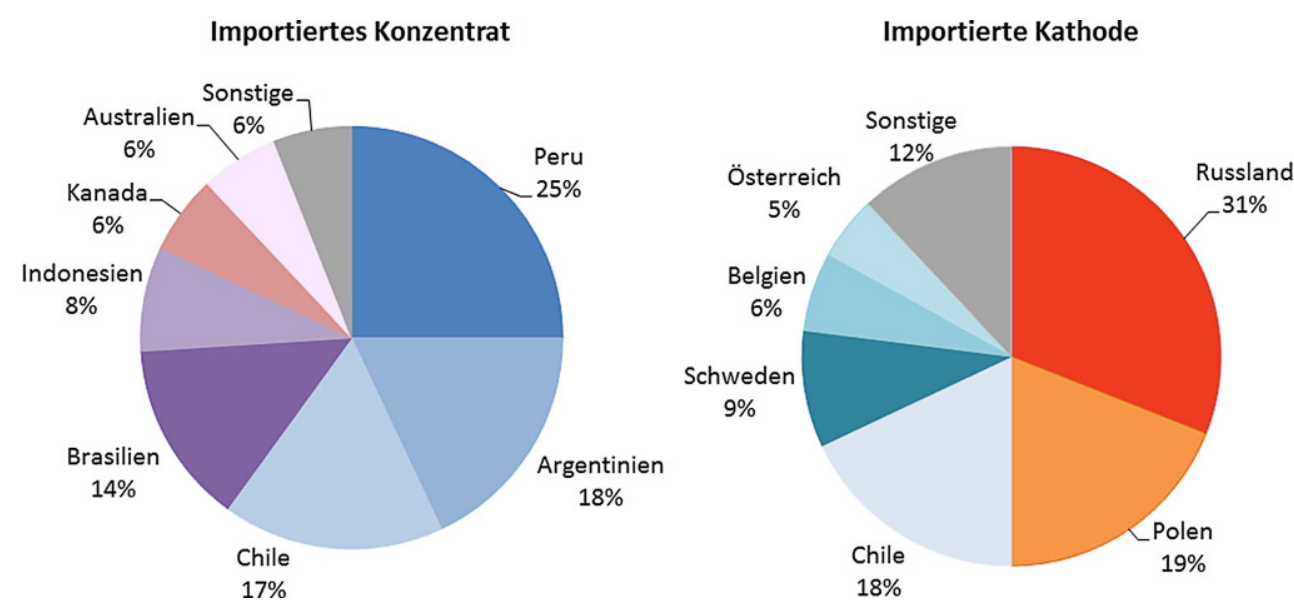
Abb. 3 Modell der Kupfergewinnung (Parameter in grauer Schrift: $E_{\text {Mix }}$ Elektrizitätserzeugung, $O G$ Erzgehalt, $P$ Anteil der jeweiligen Abbaumethode bzw. Prozessroute)

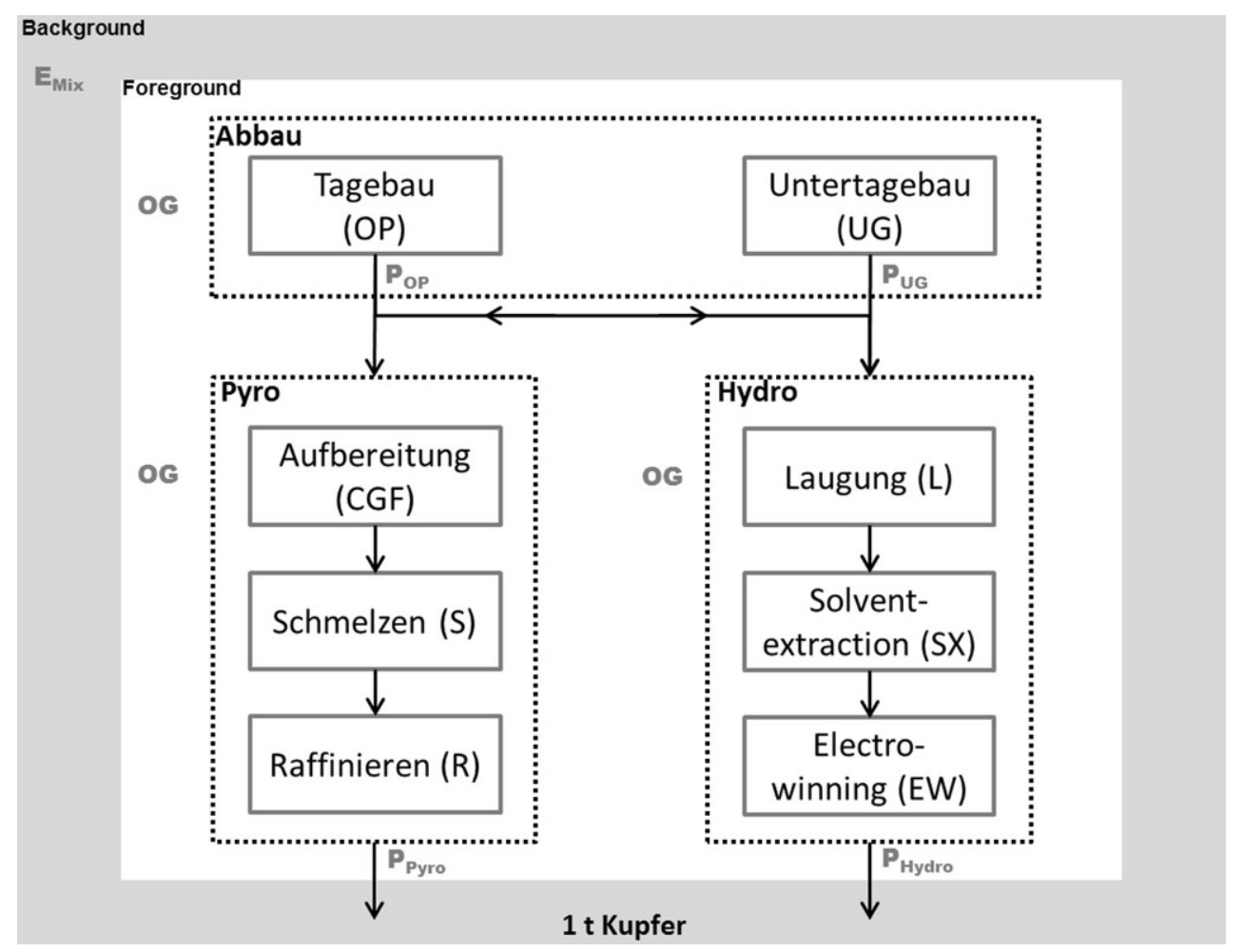

Energiebedarfs, u.a. in Abhängigkeit des Erzgehalts. Keines der Modelle liefert jedoch eine geeignete Basis zur Ermittlung des Energiebedarfs unterschiedlicher Systeme, da sie nicht detailliert genug sind oder sich auf einzelne Minen beziehen. Sicherlich sind Bewertungen einzelner Minen sehr wichtig und würden im Falle einer umfassenden und zuverlässigen Datengrundlage auch die genauesten Werte liefern. Leider ist die Datenbasis jedoch häufig unzureichend, weshalb Bewertungen nur für Einzelfälle zu finden und die vorhandenen Bewertungen z.T. ungenau sind. Ein generisches Modell, welches durch eine Modularisierung und Parametrisierung spezifisch angepasst werden kann, trägt somit zur Schließung bestehender Datenlücken bei.

Das im Rahmen dieser Arbeit eingesetzte Modell wird in Rötzer und Schmidt (2020) detailliert beschrieben. Nachfolgend wird das Modell kurz vorgestellt.

Abb. 3 zeigt eine schematische Darstellung des Modells der Kupfergewinnung. Dieses ist in ein Foregroundund Background-Modell aufgeteilt. Das Foreground-Modell enthält alle direkt an der Kupfergewinnung beteiligten Prozesse. Im Background-Modell sind alle vorgelagerten Prozesse zur Herstellung der im Foreground-Modell eingesetzten Roh-, Hilfs- und Betriebsstoffe enthalten. Um eine individuelle Anpassung des Modells zu ermöglichen, ist das Modell wie bereits erwähnt modularisiert und parametrisiert.
Im Background-Modell spielt die Elektrizitätserzeugung eine wichtige Rolle. Für die Bereitstellung von Elektrizität stehen verschiedene Technologien zur Verfügung, die mit einem unterschiedlichen Aufwand verbunden sind. Je nach Technologie muss z. T. deutlich mehr Primärenergie eingesetzt werden, als Elektrizität erzeugt wird. Zum Beispiel haben Kohlekraftwerke einen Wirkungsgrad von ca. 33\%. Auch auf die Emission von Treibhausgasen hat die Elektrizitätserzeugung einen hohen Einfluss. Werden hauptsächlich fossile Energieträger eingesetzt, dann entsteht durch deren Verbrennung Kohlenstoffdioxid $\left(\mathrm{CO}_{2}\right)$. Die Elektrizitätserzeugung $E_{M i x}$ muss daher für die einzelnen Kupferquellen, d.h. Mine bzw. Metallhütte, angepasst werden. Dies kann durch die Verwendung spezifischer Anlagen einer Mine oder Metallhütte geschehen oder bei einer Anbindung an das Stromnetz durch die Verwendung des nationalen Strommixes. Für die vorliegende Arbeit wird für die Bereitstellung elektrischer Energie der nationale Strommix angenommen. Dieser wurde für die Länder entsprechend der in Rötzer und Schmidt (2020) vorgestellten Methodik ermittelt. Die ermittelten Werte sind in Tab. 3 im Anhang zu finden. Bei nicht-energetischen Stoffen wird aufgrund deren geringfügigeren Bedeutung für den KEA (und das GWP) der Metallerzeugung (siehe z. B. ICA 2017) auf globale Durchschnittswerte zurückgegriffen. Dies gilt auch für die direkte Nutzung fossiler Energieträger.

Betrachtet man das Foreground-Modell, so sind unterschiedliche Prozessrouten möglich, weshalb das Modell in 
den Abbau und in die weiteren Verarbeitungsrouten unterteilt wird. Der Abbau kann entweder im Tagebau (OP) oder Untertagebau (UG) stattfinden. Dabei werden das Erz und der Abraum (zum Zeitpunkt des Abbaus wertloses Gestein, welches das Erz umgibt) mittels einer Sprengung gelöst und aus der Mine transportiert. Je nach Erztyp folgt eine von zwei möglichen Verarbeitungsrouten. Bei sulfidischen Erzen kommen pyrometallurgische Verfahren zum Einsatz, bei oxidischen Erzen hydrometallurgische Verfahren. Bei der pyrometallurgischen Prozessroute (Pyro) wird das Erz vor der weiteren metallurgischen Behandlung aufbereitet (beinhaltet Zerkleinern, Mahlen, Flotieren (CGF)), sodass ein Konzentrat (Kupfergehalt ca. 30\%) entsteht. Bei der hydrometallurgischen Prozessroute (Hydro) wird das Kupfer mittels Säuren aus dem grob zerkleinerten Erz gelöst. Die Lösung wird dann weiter verarbeitet, um das enthaltene Kupfer zu extrahieren. Hinsichtlich der einzelnen Prozesse wurde für alle Länder vereinfachend angenommen, dass diese den gleichen technologischen Stand aufweisen. Jedoch werden die verschiedenen Abbaumethoden (OP oder UG) und die nachfolgenden Prozessrouten (Pyro oder Hydro) über ihren Anteil $P$ an der Gesamtproduktion einbezogen und sind somit länderspezifisch anpassbar.

Innerhalb der einzelnen Prozessschritte wird der Erzgehalt $O G$ als weiterer Parameter eingepflegt, der an die spezifischen Gegebenheiten der Bezugsquelle, hier des Landes aus welchem Kupferkonzentrat bzw. Kupfer bezogen wird, angepasst werden kann. Der Erzgehalt beeinflusst den Aufwand maßgeblich. Grundsätzlich sind im Modell weitere Parameter wie die Abbautiefe oder das Erz-Abraum-Verhältnis berücksichtigt, aufgrund der mangelnden Datenverfügbarkeit wie auch ihres geringen Einflusses auf das Gesamtergebnis wurden sie für die vorliegende Arbeit mit Standardwerten hinterlegt. Die einzelnen Prozessschritte werden detailliert im Anhang beschrieben.

\subsection{Allokation}

Viele Metalle kommen vergesellschaftet im Erz vor. Das heißt, in einem Erz sind mehrere Metalle enthalten. Einige der Prozessschritte ermöglichen dann die Gewinnung mehrerer Metalle. Ihr Aufwand ist dementsprechend unter den Produkten aufzuteilen. Dies entspricht der üblichen Vorgehensweise in Ökobilanzen (LCAs) und ist unter dem Begriff der Allokation bekannt. Auch Kupfer kommt häufig vergesellschaftet mit Molybdän, Gold und Silber vor. Aber auch eine Vergesellschaftung mit z. B. Nickel ist möglich. Im Modell wird eine ökonomische Allokation vorgenommen. Dabei wird der Aufwand basierend auf dem ökonomischen Wert der enthaltenen Produkte aufgeteilt. Die Preisermittlung des Konzentrats und der darin enthaltenen Metalle resultiert aus der Nettoschmelzrendite, wie sie industrietypisch angewandt wird (Wellmer et al. 2008,
74 ff.). Die Ermittlung eines Allokationsfaktors für die anschließenden Prozessschritte (Schmelzen und Raffinieren) entspricht der klassischen Vorgehensweise einer ökonomischen Allokation. Die zugrunde gelegten Preise und Produktionsmengen sind dem Anhang (Tab. 4) zu entnehmen. Für nicht-metallische Nebenprodukte wie Schwefelsäure wird eine Gutschrift vorgenommen. Der Transportaufwand wird im Gegensatz zur Vorgehensweise beim Herstellungsaufwand vollständig Kupfer zugerechnet, da dieses als alleiniger Grund des Transports angesehen wird und auch hinsichtlich der Masse (welche den Transportaufwand neben der Strecke maßgeblich bestimmt) der enthaltenen Wertstoffe nahezu $100 \%$ ausmacht.

\subsection{Ermittlung des Gesamtaufwands}

Der Gesamtaufwand der Kupferbereitstellung für Deutschland ergibt sich aus der Summe der verschiedenen Bezugsquellen von Kupfer. Diese sind in Abb. 4 dargestellt. Beim Bezug von Konzentrat werden alle in Abb. 2 angegebenen Bezugsländer betrachtet; ihre Anteile am importierten Konzentrat werden auf $100 \%$ normiert. Gleiches gilt für raffiniertes Kupfer, dort werden aufgrund der Datenverfügbarkeit jedoch nur die drei Hauptbezugsländer Russland, Polen und Chile in die Betrachtung einbezogen und ihr Anteil jeweils auf $100 \%$ der importierten Kupferkathoden normiert. Unraffiniertes Kupfer wurde aufgrund des geringen Anteils an der gesamten Menge nicht berücksichtigt. Für jede Bezugsquelle wird das Modell über die Parameter $\left(P_{\mathrm{OP}}, P_{\mathrm{UG}}, P_{\mathrm{Hydro}}, P_{\mathrm{Pyro}}, O G, E_{\mathrm{Mix}}\right)$ angepasst und der KEA bzW. das GWP berechnet. Sofern für die Parameter keine länderspezifischen Daten verfügbar waren, wurden (auf die jährliche Produktionsmenge bezogen) repräsentative Minen in den Ländern recherchiert. Die verwendeten Werte sind Tab. 4 und 5 im Anhang zu entnehmen.

Beim Bezug von Kupferkathoden wird angenommen, dass das Kupfer auch in dem Land abgebaut wird, indem es metallurgisch behandelt wird. Teilweise wird der Metallurgie noch Kupferschrott z. B. zur Kühlung hinzugefügt. Dadurch ist auch ein geringer Anteil Sekundärkupfer in den hier berichteten Werten enthalten. Der Anteil liegt bei max. ca. 10\% (Goonan 2005; Schäfer 2021), weshalb hier von Primärkupfer gesprochen wird.

Der Transport gliedert sich in den Transport von der Mine zum Hafen per LKW und anschließend mittels Schiff nach Deutschland. Grundsätzlich kommen für den Landtransport auch andere Transportmittel wie Züge infrage. Da die Anteile der einzelnen Transportmittel am Landtransport nicht bekannt sind, wurde zur Vermeidung einer Unterschätzung des Aufwands (KEA und GWP) der Transport via LKW angenommen. Als Zielhafen für den Schiffstransport wurde Hamburg gewählt. Die länderspezi- 
Abb. 4 Modell der Bereitstellung von Primärkupfer für Deutschland

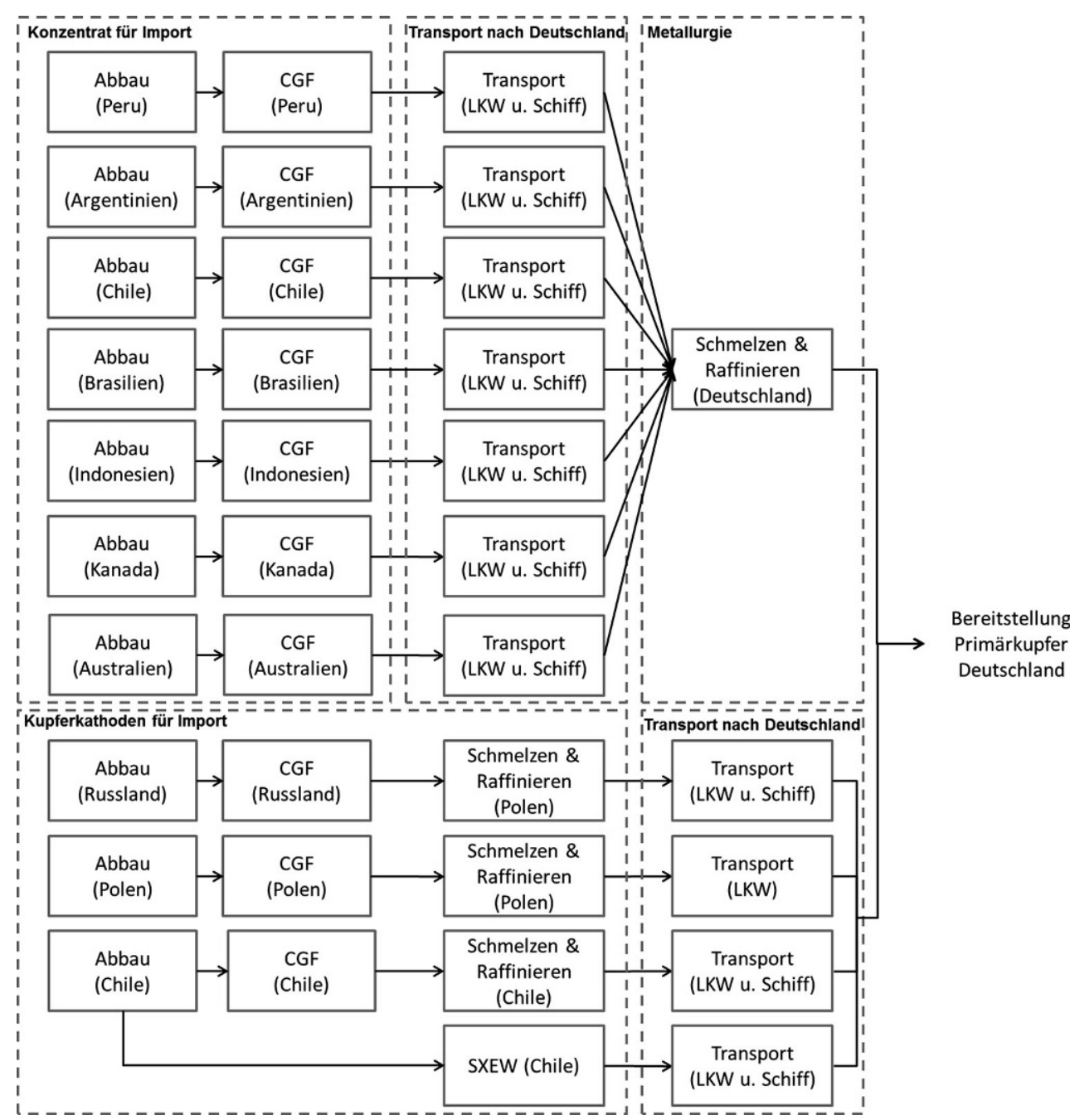

fischen Annahmen sind ebenfalls in Tab. 5 im Anhang zu finden.

\section{Ergebnisse}

Der KEA für das für Deutschland bereitgestellte Primärkupfer beträgt rund $40 \mathrm{MJ} / \mathrm{kg} \mathrm{Cu}$. Das GWP liegt bei etwa $3,3 \mathrm{~kg} \mathrm{CO}$ - $\mathrm{Äq} / \mathrm{kg} \mathrm{Cu}$.

Der KEA bzw. das GWP für importiertes Konzentrat und die anschließende metallurgische Behandlung in Deutschland liegt je nach Bezugsquelle zwischen 36 und $101 \mathrm{MJ} / \mathrm{kg} \mathrm{Cu}$ bzw. 3,0 und 7,8 kg CO $\mathrm{CO}_{2}-\ddot{\mathrm{Aq}} / \mathrm{kg} \mathrm{Cu}$ (siehe Tab. 1). In Abb. 5 ist der KEA bzw. das GWP der einzelnen Länder aufgeschlüsselt nach den Prozessschritten dargestellt. Die Reihenfolge der Länder orientiert sich dabei am Erzgehalt (aufsteigend). Die Analyse zeigt, dass der Erzgehalt der Lagerstätten wesentlich zum Aufwand beiträgt. Insbesondere die Länder, in welchen das nach Deutschland importierte Kupferkonzentrat gewonnen wird, haben alle
Erzgehalte, die unter $1 \% \mathrm{Cu}$ liegen (Argentinien, Kanada, Chile, Australien, Brasilien, Indonesien und Peru). Argentinien und Kanada haben mit $0,5 \% \mathrm{Cu}$ die geringsten Erzgehalte. Dies sowie der ressourcenintensive Strommix und die weiten Transportdistanzen führen dazu, dass das aus Argentinien stammende und in Deutschland weiterverarbeitete Konzentrat den höchsten spezifischen KEA hat. Beim GWP ist Australien aufgrund des hohen Kohleanteils an der Stromerzeugung führend. In Australien wird zudem hauptsächlich im Untertagebau abgebaut, was mit einem höheren spezifischen KEA bzw. GWP verbunden ist als der Tagebau und somit insgesamt zu einem höheren Aufwand führt. Indonesien und Peru bieten das Konzentrat mit dem niedrigsten KEA bzw. GWP. Beide Länder haben einen vergleichsweise hohen Erzgehalt. Die als repräsentativ für Indonesien angenommene Mine baut neben Kupfer auch noch große Mengen an Gold ab, welches aufgrund der Allokation einen signifikanten Teil des Aufwands trägt.

Für importierte Kupferkathoden (raffiniertes Kupfer) liegt der KEA je nach Land zwischen etwa 6 und 58 MJ/ 


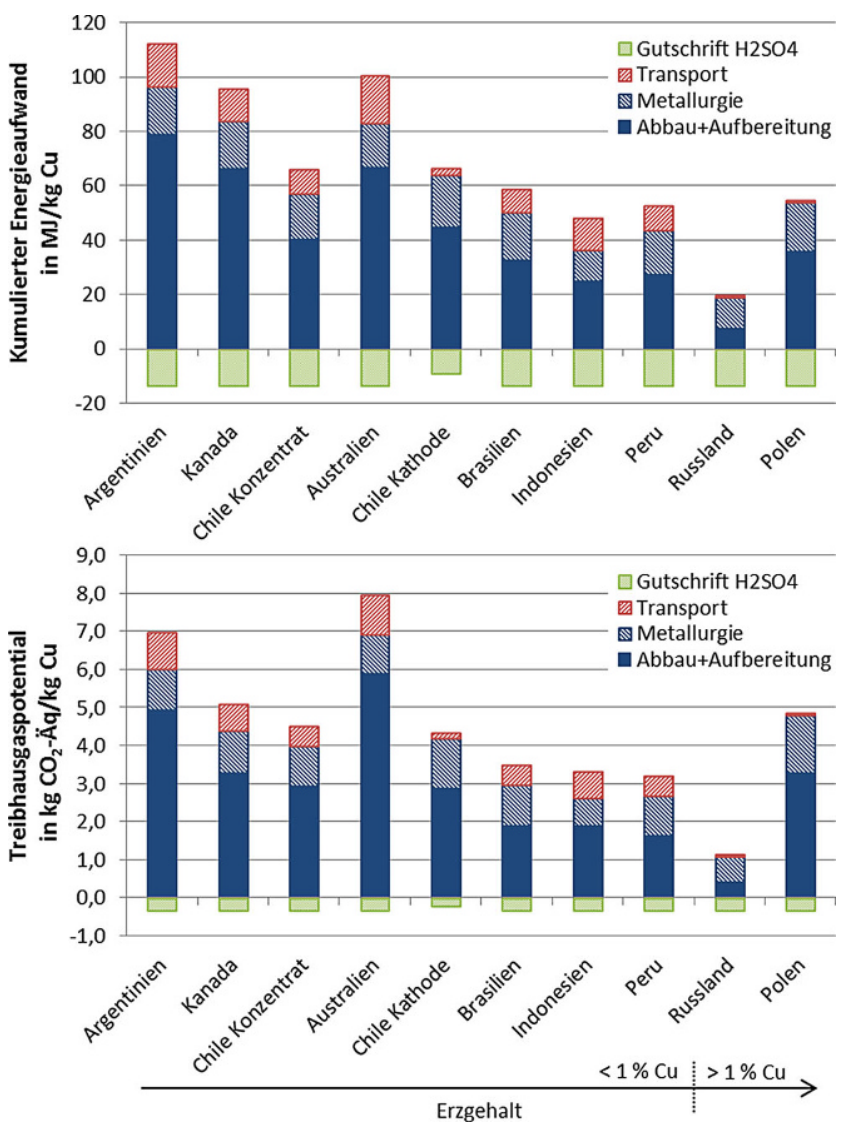

Abb. 5 KEA bzw. GWP der Kupferbereitstellung für die einzelnen Bezugsländer aufgeschlüsselt nach den Beiträgen der Prozessschritte. Die Reihenfolge der Länder orientiert sich am Erzgehalt (aufsteigend) $\mathrm{kg} \mathrm{Cu}$. Den höchsten Wert hat Chile aufgrund des im Vergleich zu Russland und Polen deutlich geringeren Erzgehalts. Auch die hydrometallurgische Kupfergewinnung, die in Chile rund $35 \%$ der Kupferherstellung ausmacht, wirkt sich ungünstig aus, da diese mit einem höheren Aufwand verbunden ist als die pyrometallurgische Verarbeitung. Dies erklärt auch, warum die aus Chile importierten Kupferkathoden einen höheren Aufwand haben als das in Deutschland aufbereitete chilenische Kupferkonzentrat. Bei Letzterem handelt es sich ausschließlich um pyrometallurgisch gewonnenes Kupfer. Mit dem geringsten Aufwand sind die aus Russland importierten Kupferkathoden verbunden. In Russland wie auch in Polen beträgt der Kupfergehalt der abgebauten Lagerstätten 1,5 bzw. 1,6\%. Dies wirkt sich positiv auf den KEA wie auch das GWP aus. Russland hat jedoch einen relativ hohen Anteil an Kernenergie (16\%), was zu einem geringen GWP im Vergleich zu Polen führt. Polens Elektrizitätserzeugung basiert im Jahr 2010 zu $93 \%$ auf fossilen Energieträgern, hauptsächlich Kohle, und ist somit mit hohen Treibhausgasemissionen verbunden. Sowohl Russland als auch Polen nutzen beide den Untertagebau. Darin liegt auch der höhere Energiebedarf Polens im Vergleich zu anderen Ländern begründet, die einen deutlich niedrigeren Erzgehalt haben. Für Russland ist begünstigend, dass Kupfer dort gemeinsam mit Nickel abgebaut wird, welchem ein hoher Anteil des Aufwands zugerechnet wird, da sein ökonomischer Wert deutlich über dem von Kupfer liegt. Aufgrund des hohen Anteils des aus Russland importierten Kupfers an der Kupferbereitstellung für Deutschland wirken sich die errechneten Werte für Russland relativ stark auf das Gesamtergebnis aus. Läge das (allozierte) GWP bzw. der KEA der aus Russland importierten Kathoden auf ähnlichem Niveau wie Polen oder Chile $\left(40 \mathrm{MJ} / \mathrm{kg} \mathrm{Cu}\right.$ und $\left.4 \mathrm{~kg} \mathrm{CO} \mathrm{CO}_{2}-\ddot{\mathrm{Aq}} / \mathrm{kg} \mathrm{Cu}\right)$, würde sich

Tab. 1 KEA und GWP für nach Deutschland importiertes Kupfer bzw. Kupferkonzentrat nach Ländern

\begin{tabular}{llll}
\hline Importiertes Gut & Land & KEA & GWP \\
Konzentrat & & MJ/kg Cu & $3,0(3,7)$ \\
& Peru & $40(60)$ & $6,8(7,2)$ \\
& Argentinien & $101(116)$ & $4,3(4,9)$ \\
& Chile & $54(72)$ & $3,3(3,6)$ \\
& Brasilien & $46(60)$ & $3,1(5,2)$ \\
& Indonesien & $36(74)$ & $4,9(5,4)$ \\
Kaffiniertes Cu (Kathoden) & Kanada & $84(100)$ & $7,8(8,8)$ \\
& Australien & $89(111)$ & $0,9(2,9)$ \\
Gesamt Cu aus importiertem Konzentrat & Russland & $6(51)$ & $4,6(5,0)$ \\
Gesamt raffiniertes Cu & Polen & $42(56)$ & $4,1(4,6)$ \\
Gesamt & Chile & $58(70)$ & $4,4(5,1)$ \\
\hline
\end{tabular}

Die angegebenen Werte beziehen sich auf die Bereitstellung von $1 \mathrm{~kg}$ raffiniertes Kupfer für Deutschland In Klammern sind die nicht allozierten Werte angegeben 
das Gesamtergebnis auf $51 \mathrm{MJ} / \mathrm{kg} \mathrm{Cu}$ bzw. $4,3 \mathrm{~kg} \mathrm{CO}_{2}-$ $\ddot{A} q / \mathrm{kg} \mathrm{Cu}$ erhöhen.

Im Vergleich zwischen den importierten Konzentraten und Kathoden fällt auf, dass der Transportaufwand für Konzentrat aufgrund des geringeren Metallgehalts des transportierten Guts $(30 \% \mathrm{Cu})$ ca. drei- bis viermal so hoch ist wie für den Transport von raffiniertem Kupfer. Der Transportaufwand bei den Konzentraten liegt bei durchschnittlich $12 \mathrm{MJ} / \mathrm{kg} \mathrm{Cu}$ und $0,7 \mathrm{~kg} \mathrm{CO}_{2}-\mathrm{Ä} / \mathrm{kg} \mathrm{Cu}$. Eine metallurgische Behandlung in der Nähe der Minen scheint also grundsätzlich sinnvoll und würde zudem die Wertschöpfung in den Abbauländern, die meist wirtschaftlich schwächer gestellt sind, erhöhen. Hier gilt es jedoch den Einzelfall zu prüfen und weitere technische, regulatorische und rohstoffpolitische Aspekte zu berücksichtigen.

Betrachtet man die Orte, an denen der Aufwand entsteht, so fällt in Deutschland nur einen geringer Teil des KEA bzw. GWP an. Beim Import von Konzentraten sind dies die Aufwendungen für die Metallurgie. Beim Import von Kupferkathoden fallen nahezu alle Emissionen und Energieaufwände in den Ländern an, in welchen das raffinierte Kupfer hergestellt wird. Eine Ausnahme stellen hier lediglich die grenzüberschreitenden Transporte dar.

Der Anteil Deutschlands bei der Herstellung von Kupfer aus importiertem Konzentrat liegt beim KEA bzw. GWP zwischen 15 und $31 \%$ bzw. 15 und $32 \%$ und beläuft sich insgesamt auf $0,35 \mathrm{~kg} \mathrm{CO}_{2}-\mathrm{Ä} / \mathrm{kg} \mathrm{Cu}$. Dabei ist die Gutschrift für Schwefelsäure noch nicht berücksichtigt. Da die Schwefelsäure jedoch beim Schmelzen entsteht, welches in Deutschland stattfindet, trägt diese zusätzlich zur Reduktion des örtlichen Aufwands bei, wobei die entstandenen Emissionen durch die Kupferverhüttung und -raffination beinahe ausgeglichen werden. Im Hinblick auf das territoriale Ziel der Klimaneutralität führen Einsparungen von primärem Kupfer oder Optimierungen in der Prozesskette daher in erster Linie zu einer Reduktion der globalen Treibhausgasemissionen, d.h. die in Deutschland direkt verursachten Emissionen werden nur geringfügig beeinflusst.

\section{Diskussion und Schlussfolgerungen}

Mittels des vorgestellten Modells wurden der KEA sowie das GWP der Kupferbereitstellung für Deutschland ermittelt. Im Vergleich zum durchschnittlichen globalen Aufwand der Kupferherstellung, welche die International Copper Association (ICA) mit $47 \mathrm{MJ} / \mathrm{kg} \mathrm{Cu}$ und $4,1 \mathrm{~kg} \mathrm{CO}_{2-}$ $\ddot{A} q / \mathrm{kg} \mathrm{Cu}$ (ICA 2017) angibt, sind die für Deutschland ermittelten Werte um ca. 15 bzw. $20 \%$ geringer. Insbesondere der Bezug von Kupferkathoden aus Russland hat einen positiven Einfluss auf das Ergebnis, da ihr Aufwand deutlich unter dem Durchschnitt liegt, sie jedoch rund ein Drittel des nach Deutschland importierten Kupfers ausmachen.
Betrachtet man einzelne Länder, so beträgt nach Northey et al. (2013) das GWP der Kupferbereitstellung in Chile, welches global zu den bedeutendsten Kupferproduzenten gehört, zwischen 1,0 und $3,9 \mathrm{~kg} \mathrm{CO}$ - ̈̈q/t Cu. Die Chilenische Kupferkommission (Cochilco) gibt für die Jahre 2005 bis 2009 je nach Verfahren Werte zwischen 2,3 und $3,6 \mathrm{~kg} \mathrm{CO} 2-\ddot{\mathrm{A}} \mathrm{q} / \mathrm{kg} \mathrm{Cu}$ an (Cochilco 2016). Der mittels des Modells berechnete durchschnittliche Wert für Chile liegt bei 4,2 $\mathrm{CO}_{2}-\ddot{\mathrm{A}} \mathrm{q} / \mathrm{kg} \mathrm{Cu}$ (mit Allokation). Ohne Allokation beträgt der Wert 4,4 $\mathrm{kg} \mathrm{CO} \mathrm{CO}_{2}-\ddot{\mathrm{A}} \mathrm{q} / \mathrm{kg} \mathrm{Cu}$. Eine Erklärung für die leicht höheren Werte könnte der Einbezug nicht-energetischer Materialien sein, welche in den genannten Arbeiten nicht berücksichtigt wurden. Zudem beziehen sich die Werte von Northey et al. (2013) auf einzelne Minen bzw. Hütten und geben somit eine Bandbreite an, die u. a. auf unterschiedliche Verfahren und Fertigungsstufen (Konzentrat, Anode, Kathode) zurückzuführen ist.

Solche Vergleiche sind grundsätzlich schwierig, da z.B. unterschiedliche Allokationsmethoden oder Systemgrenzen angewandt werden. Einige Arbeiten (z. B. Mudd 2010; Northey et al. 2013) berichten bspw. nur den Endenergiebedarf, d.h. sie berücksichtigen die vorgelagerten Ketten wie die Elektrizitätserzeugung nicht, und können daher nicht für einen direkten Vergleich des Energieaufwands herangezogen werden. Trotzdem sind die in dieser Arbeit ermittelten Werte als realistisch einzustufen.

Unsicherheiten im Modell selbst ergeben sich durch die gemachten Annahmen, welche nachfolgend nochmals kurz diskutiert werden.

Für fast alle Länder (außer Chile) musste aufgrund fehlender durchschnittlicher Angaben zu den Parametern $\left(P_{\mathrm{OP}}\right.$, $\left.P_{\mathrm{UG}}, P_{\mathrm{Hydro}}, P_{\mathrm{Pyro}}, O G\right)$ auf Informationen zu einzelnen Minen zurückgegriffen werden. Wie durch das Modell und die Ergebnisse deutlich wird, kann u. a. der Energiebedarf aufgrund der spezifischen Eigenschaften einzelner Minen erheblich variieren. Zum Beispiel: Eine Verringerung des Erzgehalts von 1 auf 0,5\% Cu erhöht den Energiebedarf (unter sonst gleichbleibenden Bedingungen) um 100\%. Um daher sicherzustellen, dass die Ergebnisse repräsentativ sind, wurde bei der Auswahl der Minen für die genannten Länder darauf geachtet, dass diese einen hohen Anteil an der jährlichen Kupferproduktion des jeweiligen Landes haben. Dabei konnte für fast alle Länder ein Anteil von über 50\% erreicht werden. Eine Ausnahme sind hier Australien und Kanada für die jeweils knapp unter $30 \%$ der jährlichen Kupferproduktion abgedeckt sind.

Bei der Ermittlung des Aufwands von importierten Kupferkathoden wurde angenommen, dass das Kupfer im Bezugsland abgebaut, aufbereitet und metallurgisch behandelt wird. Grundsätzlich könnten auch Konzentrate aus anderen Ländern weiterverarbeitet werden. Jedoch ist zum einen der Transport von Konzentrat aufgrund seines geringen Metallgehalts im Vergleich zu raffiniertem Kupfer mit höhe- 
ren energetischen und somit auch ökonomischen Aufwänden verbunden, weshalb Unternehmen darauf bedacht sind, Transporte zu reduzieren. Zum anderen zeigt sich am Beispiel Chile, dass die Annahme, dass Konzentrate häufig im Abbauland auch weiter verarbeitet werden, durchaus plausibel ist, da dort kaum importiertes Konzentrat behandelt wird. Auch in anderen Ländern bzw. den ausgewählten Minen stimmen die Produktionszahlen von Konzentrat und Anoden bzw. Kathoden annähernd überein (KGHM 2011, S. 109f.; Norilsk Nickel 2011, S. 57 ff.).

Weitere Annahmen, die im Rahmen der vorliegenden Studie gemacht wurden, betreffen die eingesetzten Technologien für die Foreground-Prozesse sowie die Bereitstellung der Elektrizität. Für die Foreground-Prozesse, d.h. direkt an der Metallgewinnung beteiligten Prozesse, wurde für alle Länder der gleiche Stand der Technologie angenommen. Sicherlich liegen hier Unterschiede vor, die u.a. vom Entwicklungsstand und den gesetzlichen Rahmenbedingungen des Landes abhängen. Des Weiteren wurde im Modell angenommen, dass die Elektrizität aus dem nationalen Stromnetz bezogen wird. Tatsächlich sind nicht alle Minen an die nationale Energieversorgung angeschlossen. Besonders in abgelegenen Regionen ist eine Eigenversorgung üblich. Leider sind hierzu nur bedingt Informationen auf Länderebene verfügbar. Insgesamt kann aber angenommen werden, dass der nationale Strommix eine gute Schätzung der durchschnittlichen Energieerzeugungsmethoden vermittelt. Bei der Bewertung einzelner Minen jedoch sollte die tatsächliche Stromerzeugung wie auch das Technologieniveau in das Modell integriert werden.

Hinsichtlich des Bewertungsansatzes wurde die Energie als zentraler Indikator gewählt, da sie als einschränkender Faktor für die Verfügbarkeit von Rohstoffen betrachtet werden kann und stellvertretend für ökonomische als auch ökologische Aspekte steht. Zusätzlich wurden die Treibhausgasemissionen aufgrund ihrer hohen gesellschaftlichen Relevanz berechnet. Grundsätzlich könnte nun entgegnet werden, dass auch die Energie durch die Realisierung einer regenerativen Energieerzeugung in Zukunft kein knappes Gut mehr darstellen wird. Bis global eine rein regenerative Energieerzeugung erreicht wird, wird noch einige Zeit vergehen. Die International Energy Agency (IEA) geht für 2040 immer noch von einem Anteil von mindestens $50 \%$ fossiler Energieträger an der Elektrizitätserzeugung aus. Der Bergbau selbst ist nicht zwangsweise an die nationale Stromerzeugung angeschlossen, d.h. die Minen und Hütten haben eigene Anlagen zur Energieerzeugung. Bislang beschränkt sich der Einsatz erneuerbarer Energien dabei jedoch hauptsächlich auf einzelne Vorzeigeprojekte und ist noch nicht industrieweit umgesetzt. Die Potenziale jedoch, die sich durch die Nutzung regenerative Energien im Bergbau hinsichtlich des KEA und GWP ergeben, sind, wie Rötzer und Schmidt (2020) zeigen, enorm. So kann unter der Annahme des Einsatzes von Windkraft das GWP in etwa halbiert werden. Um diese Potenziale umzusetzen, müssen die richtigen Anreize und Rahmenbedingungen geschaffen werden, welche die Attraktivität einer regenerativen Stromerzeugung im Bergbau fördern (Maennling und Toledano 2018, 13, $80 \mathrm{ff}$.).

Deutschland hat auf die Energiebereitstellung im Bergbau keinen direkten Einfluss, da in Deutschland selbst kein Abbau stattfindet und die Elektrizitätsbereitstellung bei den Ländern bzw. Betreibern der Minen liegt. Auch eine Optimierung der in Deutschland angewandten Prozesse und Technologien zur Erzeugung von Kupfer aus den importierten Konzentraten kann den Gesamtaufwand aufgrund des geringen Anteils der Prozesse an diesem nur minimal reduzieren. Durchschnittlich entfallen über $60 \%$ des KEAs sowie GWPs der pyrometallurgischen Kupfergewinnung auf den Bergbau und die Aufbereitung (ohne Berücksichtigung von Nebenprodukten) (Rötzer und Schmidt 2020; ICA 2017). Dies bestätigen auch die vorliegenden Ergebnisse. Eine Senkung des KEAs der Verhüttung und Raffination in Deutschland um 20\%, senkt das Gesamtergebnis nur um 3\%. Beim GWP führt eine $20 \%$ ige Reduktion zu einer Senkung des Gesamtergebnisses um $2 \%$. Dies zeigt, dass es von zentraler Bedeutung ist, dass am Rohstoffmarkt Druck aufgebaut wird, indem der Carbon Footprint von Metallen spezifisch ausgewiesen wird und Angebote mit geringerem Carbon Footprint bevorzugt werden. Dazu müssten die Kunden aber bereit sein, höhere Preise für emissionsärmeres Kupfer zu zahlen.

Die Herausforderungen, die hinsichtlich der Rohstoffverfügbarkeit und der globalen Erderwärmung gestellt werden, können letztendlich nur durch eine Betrachtung und Optimierung der gesamten Wertschöpfungskette bewältigt werden. Dazu kann ein prozessbasiertes und parametrisiertes Modell, wie es in dieser Arbeit entwickelt wurde, beitragen. Es bietet die Möglichkeit, schnell und zuverlässig den energetischen Aufwand und die verursachten Treibhausgasemissionen der Metallgewinnung unter Berücksichtigung spezifischer Gegebenheiten zu ermitteln. Somit können u.a. Hotspots in den Lieferketten und Prozessen identifiziert und Vergleiche zwischen verschiedenen Bezugsalternativen vorgenommen werden. Eine Erweiterung um weitere Indikatoren ist grundsätzlich denkbar und bleibt für die weitere Forschung offen.

In der vorliegenden Arbeit wurde die Kupferbereitstellung für Deutschland betrachtet. Das Modell wurde somit länderspezifisch angewandt. Um detailliertere oder auch minenspezifische Bewertungen durchführen zu können, ist der Einbezug weiterer Parameter wie des Technologieniveaus oder des Abbauverfahrens im Untertagebau notwendig (siehe dazu auch Rötzer und Schmidt 2020). 


\section{Anhang}

\section{Beschreibung der einzelnen Module}

Nachfolgend werden die einzelnen Module des Modells detaillierter beschrieben. Die dazu angegebenen Formeln beziehen sich auf den KEA, sind jedoch auch für die Ermittlung des GWP gültig. Die Stoff- und Energieströme der einzelnen Prozessschritte, auf deren Basis der KEA bzw. das GWP berechnet werden, sind Rötzer und Schmidt (2020) zu entnehmen und basieren größtenteils auf Literaturwerten.

\section{Abbau}

Der Abbau kann in die Prozessschritte Bohren und Sprengen, Zerkleinern, Laden (und Transportieren) und Transportieren unterteilt werden. Untertagebauminen müssen zusätzlich noch belüftet werden, was meist mit einem hohen Energiebedarf verbunden ist. Ein zu beachtender Parameter ist das Stripping Ratio $S R^{1}$. Dieses gibt das Abraum-ErzVerhältnis an, welches beim Tagebau bis zu 10t Abraum pro Tonne abgebautem Erz betragen kann. Im Untertagebau ist dieses eher zu vernachlässigen (ca. 0,1) (Atlas Copco 2007). Das Stripping Ratio $S R$ wirkt sich abgesehen von der Ventilation auf alle Prozessschritte aus, da alle Prozessschritte nicht nur für das Erz, sondern auch für den Abraum anfallen, der ebenfalls aus der Erdkruste gelöst und meist grob zerkleinert wird, um aus der Mine transportiert zu werden. Das heißt, die erfassten Bedarfe an Hilfs- und Betriebsstoffen bzw. deren KEA müssen mit $(1+S R)$ multipliziert werden, bevor sie durch den Erzgehalt $O G$ geteilt werden, um den KEA pro Masseneinheit Metall zu erfassen. Alle nachfolgenden Prozesse unterliegen Verlusten, die über deren Effizienz $R$ einbezogen werden. Somit kann der KEA des Abbaus $E_{\mathrm{OP} \text { oder Ug }}$ pro Masseneinheit Metall wie folgt berechnet werden:

$E_{\mathrm{OP}}=\frac{\left(E_{D+B}+E_{C_{1}}+E_{T}+E_{\mathrm{Ld}}\right) \cdot(1+S R)}{O G \cdot R_{\text {Pyro oder Hydro }}}$

Mit:

$\begin{array}{ll}E_{\mathrm{OP}} & \text { KEA Tagebau in MJ/kg Cu } \\ E_{\mathrm{D}+\mathrm{B}} & \text { KEA Bohren und Sprengen in MJ/kg Erz } \\ E_{\mathrm{Ld}} & \text { KEA Laden in MJ/kg Erz } \\ E_{\mathrm{T}} & \text { KEA Transportieren in MJ/kg Erz } \\ E_{\mathrm{c}_{i}} & \text { KEA } i \text {-ter Zerkleinerungsschritt in MJ/kg Erz } \\ S R & \text { Stripping Ratio } \\ O G & \text { Erzgehalt }\end{array}$

${ }^{1}$ Im Originalmodell ist zusätzlich die Tiefe als Parameter modelliert, diese wurde ebenfalls als fixer Wert mit $500 \mathrm{~m}$ (OP und UG) angenommen.
$R_{\text {Pyro oder Hydro }}$ Effizienz der nachfolgenden Bearbeitungsroute Pyro bzw. Hydro

$E_{\mathrm{UG}}=\frac{\left(E_{D+B}+E_{L+T}+E_{C_{1}}+E_{T}\right) \cdot(1+S R)+E_{V}}{O G \cdot R_{\text {Pyro oder Hydro }}}$

Mit:

$E_{\mathrm{UG}} \quad$ KEA Untertagebau in $\mathrm{MJ} / \mathrm{kg} \mathrm{Cu}$

$E_{\mathrm{L}+\mathrm{T}} \quad$ KEA Laden und Transportieren in $\mathrm{MJ} / \mathrm{kg}$ Erz

$\mathrm{E}_{\mathrm{V}} \quad$ KEA Ventilation in MJ/kg Erz

\section{Pyrometallurgische Route}

Aufbereitung Ist das abgebaute Erz sulfidischer Art, wird es anschließend zu einem Konzentrat mit einem Kupferanteil von ca. $30 \%$ aufbereitet. Dazu wird es nochmals grob zerkleinert und anschließend gemahlen. Danach folgt eine Flotation, bei der das fein gemahlene und mit Wasser vermischte Erz mit Chemikalien versetzt wird, welche die wertvollen Mineralien „sammeln“ und an die Oberfläche transportieren, wo sie sich als Schaum absetzen und abgeschöpft werden können. Der KEA der Aufbereitung $E_{\text {CGF }}$ wird maßgeblich durch den Schritt des Mahlens bestimmt, welcher mit einem hohen Energiebedarf verbunden ist. Wie auch schon beim Abbau bestimmt der Erzgehalt $O G$ die zu verarbeitende Menge an Erz, um eine Masseneinheit Kupfer zu erzeugen. Ebenfalls ist die Effizienz $R$ der Aufbereitung und der nachfolgenden Prozessschritte zu beachten:

$E_{\mathrm{CGF}}=\frac{E_{C_{2}}+E_{G}+E_{F}}{O G \cdot R_{\mathrm{Pyro}}}=\frac{E_{C_{2}}+E_{G}+E_{F}}{O G \cdot R_{\mathrm{CGF}} \cdot R_{S} \cdot R_{R}}$

Mit:

$E_{\mathrm{CFG}} \quad$ KEA Aufbereitung in $\mathrm{MJ} / \mathrm{kg} \mathrm{Cu}$

$E_{\mathrm{G}} \quad$ KEA Mahlen in MJ/kg Erz

$E_{\mathrm{F}} \quad$ KEA Flotation in MJ/kg Erz

$R_{\mathrm{CGF}} \quad$ Effizienz Aufbereitung

$R_{\mathrm{S}} \quad$ Effizienz Schmelzen

$R_{\mathrm{R}} \quad$ Effizienz Raffination

Schmelzen und Raffinieren Das aus der Aufbereitung resultierende Konzentrat wird anschließend geschmolzen (und konvertiert), um Verunreinigungen wie Schwefel zu entfernen. Dazu wird neben fossilen Energieträgern zur Erzeugung der notwendigen Startenergie auch Sauerstoff eingesetzt. Um die Reinheit des Metalls weiter zu erhöhen, wird es abschließend raffiniert. Produkt ist eine Kupferkathode, welche aus annähernd $100 \% \mathrm{Cu}$ besteht. Da das eingehende Konzentrat meist eine ähnliche Zusammensetzung auf- 
weist, sind die Prozessschritte Schmelzen und Raffinieren nicht vom Erzgehalt abhängig, hier ist lediglich die Effizienz $R$ zu beachten:

$$
E_{S+R}=\frac{E_{S}}{R_{S} \cdot R_{R}}+\frac{E_{R}}{R_{R}}
$$

Mit:

$$
\begin{array}{ll}
E_{\mathrm{S}+\mathrm{R}} & \text { KEA Schmelzen und Raffinieren in } \mathrm{MJ} / \mathrm{kg} \mathrm{Cu} \\
E_{\mathrm{S}} & \text { KEA Schmelzen in } \mathrm{MJ} / \mathrm{kg} \mathrm{Cu} \text {-Input } \\
E_{\mathrm{R}} & \text { KEA Raffination in } \mathrm{MJ} / \mathrm{kg} \mathrm{Cu} \text {-Input }
\end{array}
$$

\section{Hydrometallurgische Route}

Laugung Oxidische Erze werden zuerst grob zerkleinert und dann mittels Säuren, häufig Schwefelsäure, gelaugt. Dazu wird das angehäufte Erz mit Säure berieselt. Resultat ist eine kupferhaltige Lösung. Der Einsatz an Säure und Energie für die Zerkleinerung und Laugung wird durch die bearbeitete Menge an Erz bestimmt. Damit sind der Erzgehalt $O G$ sowie die Effizienz $R$ zu berücksichtigen, um den KEA der Laugung $E_{\mathrm{L}}$ pro Masseneinheit gewonnenes Kupfer zu berechnen:

$$
E_{L}=\frac{E_{C_{2}}+E_{\mathrm{Leach}}}{O G \cdot R_{\mathrm{Hydro}}}=\frac{E_{C_{2}}+E_{\mathrm{Leach}}}{O G \cdot R_{L} \cdot R_{\mathrm{SX}} \cdot R_{\mathrm{EW}}}
$$

Mit:
$E_{\mathrm{L}}$
$E_{\text {Leach }}$
$R_{\mathrm{SX}}$
KEA Laugung gesamt in $\mathrm{MJ} / \mathrm{kg} \mathrm{Cu}$
KEA Laugen in MJ/kg Erz
$R_{\mathrm{EW}}$
Effizienz Solventextraktion
Effizienz Gewinnungselektrolyse

SX-EW Das Kupfer wird durch eine Solventextraktion (SX) aus der Lösung extrahiert und anschließend mittels einer Gewinnungselektrolyse (EW) gewonnen. Diese Prozessschritte sind entsprechend der Pyrometallurgie nicht vom Erzgehalt abhängig und können wie folgt berechnet werden:

$$
E_{\mathrm{SX}+\mathrm{EW}}=\frac{E_{\mathrm{SX}}}{R_{\mathrm{SX}} \cdot R_{\mathrm{EW}}}+\frac{E_{\mathrm{EW}}}{R_{\mathrm{EW}}}
$$

Mit:

$$
\begin{array}{ll}
E_{\mathrm{SX}+\mathrm{EW}} & \text { KEA SX-EW in } \mathrm{MJ} / \mathrm{kg} \mathrm{Cu} \\
E_{\mathrm{SX}} & \text { KEA Solventextraktion in } \mathrm{MJ} / \mathrm{kg} \mathrm{Cu}-\mathrm{Input} \\
E_{\mathrm{SX}} & \text { KEA Gewinnungselektrolyse in } \mathrm{MJ} / \mathrm{kg} \mathrm{Cu}- \\
& \text { Input }
\end{array}
$$

Für das SR sowie die Effizienz der Prozessschritte wurden standardisierte Werte basierend auf Literaturangaben angenommen. Die Werte diese sind Tab. 2 zu entnehmen.
Tab. 2 Effizienz und Stripping Ratio der Prozessschritte. (Schlesinger et al. 2011; Atlas Copco 2007)

\begin{tabular}{ll}
\hline Prozess & Effizienz R [\%] \\
Aufbereitung (CGF) & 90 \\
Schmelzen (S) & 97 \\
Raffinieren (R) & 100 \\
Laugung (L) & 70 \\
Solventextraktion (SX) & 90 \\
Gewinnungselektrolyse (EW) & 100 \\
Prozess & Stripping Ratio \\
Tagebau & 2,5 \\
Untertagebau & 0,1 \\
\hline
\end{tabular}

Tab. 3 KEA und GWP der Elektrizitätserzeugung verschiedener Länder. (Zur Methodik und für detailliertere Informationen siehe Rötzer und Schmidt 2020)

\begin{tabular}{lll}
\hline Land & $\begin{array}{l}\text { KEA } \\
{[\mathrm{MJ} / \mathrm{kWh}]}\end{array}$ & $\begin{array}{l}\text { GWP } \\
{[\mathrm{kg} \mathrm{CO} 2-\mathrm{Ä} / \mathrm{kWh}]}\end{array}$ \\
\hline Chile & 7,8 & 0,52 \\
Argentinien & 9,1 & 0,45 \\
Australien & 10,0 & 0,88 \\
Peru & 6,2 & 0,26 \\
Kanada & 6,5 & 0,22 \\
Indonesien & 9,4 & 0,72 \\
Brasilien & 4,9 & 0,11 \\
Polen & 10,4 & 0,98 \\
Russland & 8,6 & 0,43 \\
Deutschland & 9,4 & 0,56 \\
\hline
\end{tabular}




\section{Weitere Informationen und Annahmen}

Tab. 4 Produktionsmengen Haupt- und Nebenprodukte

\begin{tabular}{|c|c|c|c|c|c|c|c|c|c|c|c|}
\hline \multirow[t]{2}{*}{ Land, Mine } & \multirow{2}{*}{$\begin{array}{l}\text { Produktion } \\
{[\mathrm{kt}} \\
\mathrm{Cu}]^{\mathrm{a}}\end{array}$} & \multicolumn{7}{|c|}{$\begin{array}{l}\text { Produktion Nebenprodukte } \\
{[\mathrm{t}]}\end{array}$} & \multirow{2}{*}{\multicolumn{2}{|c|}{$\begin{array}{l}\text { Allokation } \mathrm{Cu} \\
{[\%]} \\
\text { KonzentratMetall }\end{array}$}} & \multirow[t]{2}{*}{ Quelle $^{b}$} \\
\hline & & $\overline{\text { Mo }}$ & $\mathrm{Au}$ & $\mathrm{Ag}$ & $\mathrm{Zn}$ & $\mathrm{Pb}$ & $\mathrm{Ni}$ & PGM & & & \\
\hline Peru, Antamina & $\begin{array}{l}779 \\
(>60 \%)\end{array}$ & 2405 & - & 434 & 401.183 & 8876 & - & - & 83 & - & BHP (2010), Jahr 2010 \\
\hline $\begin{array}{l}\text { Argentinien, } \\
\text { Alumbrera }\end{array}$ & $\begin{array}{l}146 \\
(100 \%)\end{array}$ & - & - & 14 & - & - & - & - & 99 & - & $\begin{array}{l}\text { Northey (2018); Nor- } \\
\text { they et al. (2013), } \\
\text { Mittelwert 2007-2012 }\end{array}$ \\
\hline Chile & 3600 & 42.000 & 17 & 862 & - & - & - & - & 92 & 96 & Cochilco $(2016,2015)$ \\
\hline $\begin{array}{l}\text { Brasilien, Sosse- } \\
\text { go }\end{array}$ & $\begin{array}{l}112 \\
(>50 \%)\end{array}$ & - & - & - & - & - & - & - & 100 & - & $\begin{array}{l}\text { Vale (o.J.), Mittelwert } \\
\text { 2005-2016 }\end{array}$ \\
\hline $\begin{array}{l}\text { Indonesien, Gras- } \\
\text { berg }\end{array}$ & $\begin{array}{l}540 \\
(>60 \%)\end{array}$ & - & 56 & - & - & - & - & - & 58 & - & $\begin{array}{l}\text { Freeport-McMoRan } \\
\text { Copper und Gold } \\
\text { (2011), Jahr } 2010\end{array}$ \\
\hline $\begin{array}{l}\text { Kanada, Highland } \\
\text { Valley }\end{array}$ & $\begin{array}{l}165 \\
(<30 \%)\end{array}$ & 2850 & - & - & - & - & - & - & 96 & - & $\begin{array}{l}\text { Northey et al. (2013), } \\
\text { Mittelwert 2004-2007 }\end{array}$ \\
\hline $\begin{array}{l}\text { Australien, Ernest } \\
\text { Henry Mt. Isa, } \\
\text { Townsville }\end{array}$ & $\begin{array}{l}257 \\
(<30 \%)\end{array}$ & - & 3 & - & - & - & - & - & 92 & - & $\begin{array}{l}\text { Northey et al. (2013), } \\
\text { Mittelwert 2005-2012 }\end{array}$ \\
\hline $\begin{array}{l}\text { Russland, Norni- } \\
\text { ckel Russia }\end{array}$ & $\begin{array}{l}366 \\
(>50 \%)\end{array}$ & - & - & - & - & - & 235.518 & 105 & 24 & 67 & $\begin{array}{l}\text { Norilsk Nickel (2011), } \\
\text { Jahr 2010, Polar Divisi- } \\
\text { on und Kola MMC }\end{array}$ \\
\hline Polen, KGHM & $\begin{array}{l}701 \\
(>100 \%)\end{array}$ & - & 0,8 & 1161 & - & 42.900 & - & - & 97 & 99 & $\begin{array}{l}\text { KGHM (2011), Jahr } \\
\text { 2010, Smelter Produkti- } \\
\text { on }\end{array}$ \\
\hline
\end{tabular}

Für die Preise wurde der Durchschnittswert der Jahre 2005-2015 basierend auf Kelly und Matos (2014) angenommen

Für Schwefelsäure wird ein pauschaler Wert für die Produktion von $2 \mathrm{~kg}$ Schwefelsäure pro $\mathrm{kg}$ Kupfer angenommen und die entsprechenden Aufwände gutgeschrieben (Cochilco 2015)

${ }^{a}$ In Klammern ist der Anteil der Mine an der jährlichen Produktion angegeben

bNorthey et al. (2013); Daten wurde dankenswerterweise von Stephen Northey bereitgestellt 


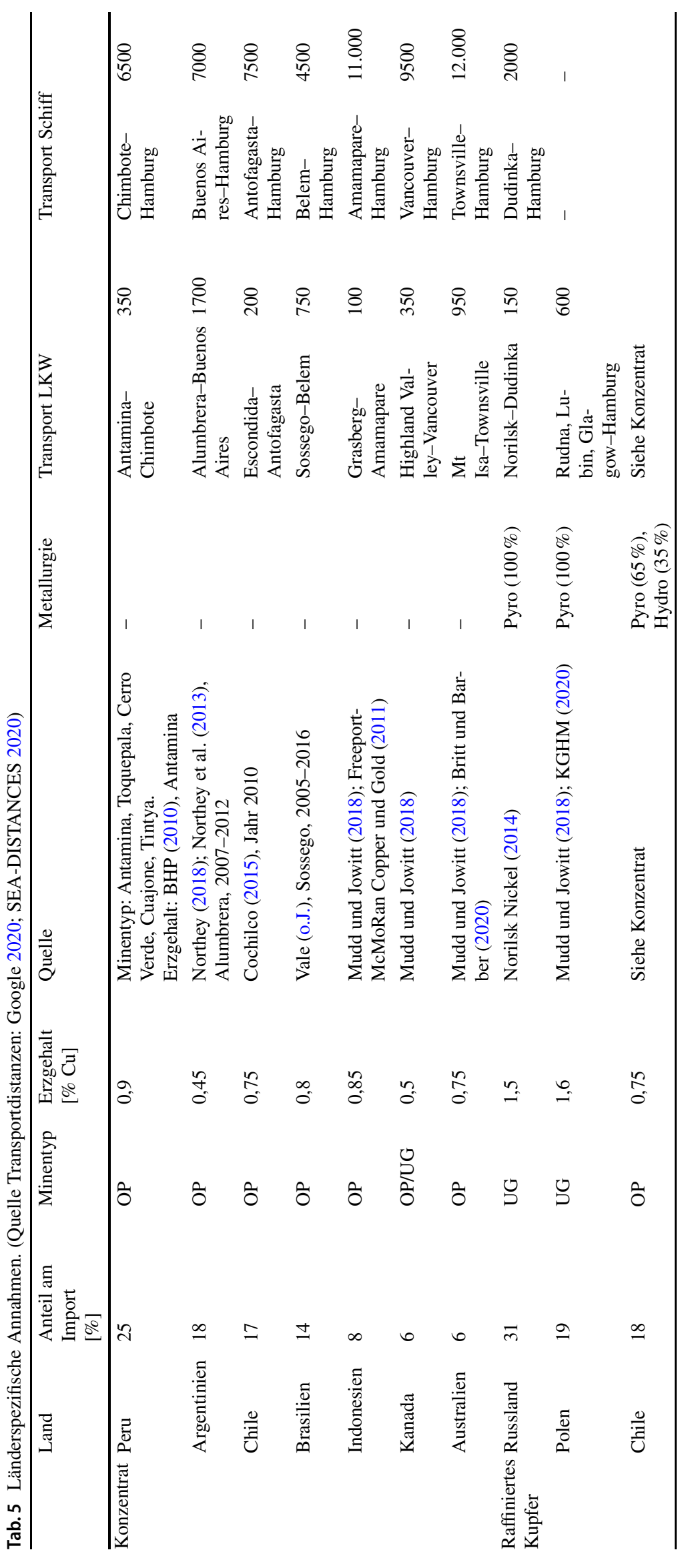


Förderung Die Dissertation und die daraus vorgestellten Ergebnisse wurden im Rahmen des kooperativen Promotionskollegs Energiesysteme und Ressourceneffizienz (ENRES) erstellt, welches von der Landesgraduiertenstiftung und des Ministeriums für Wissenschaft, Forschung und Kunst Baden-Württemberg gefördert wurde.

Funding Open Access funding enabled and organized by Projekt DEAL.

Interessenkonflikt N. Rötzer gibt an, dass kein Interessenkonflikt besteht.

Open Access Dieser Artikel wird unter der Creative Commons Namensnennung 4.0 International Lizenz veröffentlicht, welche die Nutzung, Vervielfältigung, Bearbeitung, Verbreitung und Wiedergabe in jeglichem Medium und Format erlaubt, sofern Sie den/die ursprünglichen Autor(en) und die Quelle ordnungsgemäß nennen, einen Link zur Creative Commons Lizenz beifügen und angeben, ob Änderungen vorgenommen wurden.

Die in diesem Artikel enthaltenen Bilder und sonstiges Drittmaterial unterliegen ebenfalls der genannten Creative Commons Lizenz, sofern sich aus der Abbildungslegende nichts anderes ergibt. Sofern das betreffende Material nicht unter der genannten Creative Commons Lizenz steht und die betreffende Handlung nicht nach gesetzlichen Vorschriften erlaubt ist, ist für die oben aufgeführten Weiterverwendungen des Materials die Einwilligung des jeweiligen Rechteinhabers einzuholen.

Weitere Details zur Lizenz entnehmen Sie bitte der Lizenzinformation auf http://creativecommons.org/licenses/by/4.0/deed.de.

\section{Literatur}

Atlas Copco (2007) Mining methods. In: Underground mining, 2. Aufl. Atlas Copco Rock Drills, Örebro

BGR (2012) BGR-Datenbank. Bundesanstalt für Geowissenschaften und Rohstoffe (BGR), Hannover

BGR (2020) Deutschland - Rohstoffsituation 2019. Bundesanstalt für Geowissenschaften und Rohstoffe (BGR), Hannover

BHP (2010) United States Securities and Exchange Comission FORM 20-F. Annual report pursuant to section 13 or 15 (d) of the Securities Exchange Act of 1934. BHP Group Limited und BHP Group Plc, Melbourne, London

BMWi (2019) Rohstoffstrategie der Bundesregierung. Sicherung einer nachhaltigen Rohstoffversorgung Deutschlands mit nichtenergetischen mineralischen Rohstoffen. Bundesministerium für Wirtschaft und Energie (BMWi), Berlin

Britt A, Barber J (2020) Australian Minerals Resource Assessment 2013. Copper. https://www.ga.gov.au/data-pubs/data-andpublications-search/publications/australian-minerals-resourceassessment/copper. Zugegriffen: 22. Juli 2020 (Hg. v. Geoscience Australia)

Calvo G, Mudd G, Valero A, Valero A (2016) Decreasing Ore Grades in Global Metallic Mining: A Theoretical Issue or a Global Reality? Resources 5(4):36. https://doi.org/10.3390/resources5040036

Chapman (1973) The Energy Costs of Producing Copper and Aluminium from Primary Sources. Open University, Energy Research Group, Milton Keynes

Chapman PF, Roberts F (1983) Metal resources and energy. Butterworths monographs in materials. Butterworths, London

Cochilco (2015) Aurario Estadisticas del Cobre y Otros Minerales 1995-2014. https://www.cochilco.cl/Paginas/Estadisticas/ Publicaciones/Anuario.aspx. Zugegriffen: 3. Juni 2020 (Hg. v. Chilean Copper Commission (Cochilco). Santiago de Chile)

Cochilco (2016) Aurario Estadisticas del Cobre y Otros Minerales 1996-2015. https://www.cochilco.cl/Paginas/Estadisticas/
Publicaciones/Anuario.aspx. Zugegriffen: 3. Juni 2020 (Hg. v. Chilean Copper Commission (Cochilco). Santiago de Chile)

Dorner U (2013) Rohstoffrisikobewertung Kupfer. Deutsche Rohsstoffagentur (DERA) in der Bundesanstalt für Geowissenschaften und Rohstoffe (BGR), Berlin

Freeport-McMoRan Copper \& Gold (2011) Connecting the world. 2010 Annual report. https://s22.q4cdn.com/529358580/files/doc_ financials/annual/FCX_AR_2010.pdf. Zugegriffen: 1. Sept. 2020 (Hg. v. Freeport-McMoRan Copper \& Gold. Phoenix, Arizona)

Gaines LL (1980) Energy and Material Flows in the Copper Industry. Argonne National Labaratory, Argonne

Gerst MD (2008) Revisiting the Cumulative Grade-Tonnage Relationship for Major Copper Ore Types. Econ Geol 103(3):615-628. https://doi.org/10.2113/gsecongeo.103.3.615

Google (2020) Google Maps. https://www.google.de/maps. Zugegriffen: 15 . Sept. 2020

Goonan TG (2005): Flows of Selected Materials Associated with World Copper Smelting. Open-File Report 2004-1395. U.S. Geological Survey, Reston, Virginia

Hilgers C, Becker I (2020) Local availability of raw materials and increasing global demand-aspects of resilient resource strategies. Lokale Verfügbarkeit von Rohstoffen bei steigender globaler Nachfrage - Aspekte zu resilienten Ressourcenstrategien. World Min Surf Undergr 72(5):254-263

ICA (2017) Copper Cathode Life Cycle Assessment. International Copper Association (ICA) und thinkstep, Washington D.C.

Johnson KM, Hammastrom JM, Zientek ML, Dicken CL (2014) Estimate of undiscovered copper resources of the world, 2013. U.S. Department of the Interior, U.S. Geological Survey, Reston

Kelly TD, Matos GR (Hrsg) (2014) Historical statistics for mineral and material commodities in the United States: U.S. Geological Survey (Geological Survey Data Series 140). https://minerals. usgs.gov/minerals/pubs/historical-statistics/. Zugegriffen: 14. Juli 2020

Kesler SE, Wilkinson BH (2008) Earth's copper resources estimated from tectonic diffusion of porphyry copper deposits. Geology 36(3):255-258. https://doi.org/10.1130/G24317A.1

KGHM (2011) Annual report R 2010. KGHM Polska Miedź, Lubin

KGHM (2020) Our business. Mining and enrichment—Lubin, Rudna, Polkowice-Sieroszowice. https://kghm.com/en/our-business/ mining-and-enrichment. Zugegriffen: 21. Juli 2020 (Hg. v. KGHM Polska Miedź)

Kleijn R, Voet E van der, Kramer GJ, Oers L van, Giesen C van der (2011) Metal requirements of low-carbon power generation. Energy 36(9):5640-5648. https://doi.org/10.1016/j.energy.2011. 07.003

Maennling N, Toledano P (2018) The renewable power of the mine. Accelerating renewable energy integration. Report. Columbia Center on Sustainable Investment (CCSI), New York

Marscheider-Weidemann F, Langkau S, Hummen T, Erdmann L, Tercero Espinoza LA, Angerer G, Marwede M, Benecke S (2016) Rohstoffe für Zukunftstechnologien 2016. Deutsche Rohstoffagentur (DERA) in der Bundesanstalt für Geowissenschaften und Rohstoffe (BGR), Berlin

Marsden JO (2008) Energy efficiency and copper hydrometallurgy. In: Young CA, Taylor PR, Anderson CG, Choi Y (Hrsg) Hydrometallurgy 2008. Proceedings of the Sixth International Symposium. Society for Mining, Metallurgy, and Exploration, Littleton, Colorado, $\mathrm{S} 29-40$

Meadows DH, Meadows DL, Randers J, Behrens III, WW (1972) The limits to growth. A report for the Club of Rome's project on the predicament of mankind. 2. Aufl. Universe Books, New York

Mudd GM (2009) The sustainability of mining in Australia: Key production trends and environmental implications. Research Report No RP5. Monash University and Mineral Policy Institute, Melbourne 
Mudd GM (2010) Global trends and environmental issues in nickel mining: Sulfides versus laterites. Ore Geol Rev 38(1-2):9-26. https://doi.org/10.1016/j.oregeorev.2010.05.003

Mudd GM, Jowitt SM (2018) Growing global copper resources, reserves and production: discovery is not the only control on supply. Econ Geol 113(6):1235-1267. https://doi.org/10.5382/econgeo. 2018.4590

Mudd GM, Weng Z, Jowitt SM (2013) A Detailed Assessment of Global $\mathrm{Cu}$ Resource Trends and Endowments. Econ Geol 108(5):1163-1183. https://doi.org/10.2113/econgeo.108.5.1163

Norgate T, Jahanshahi S (2010) Low grade ores-Smelt leach or concentrate? Miner Eng 23(2):65-73. https://doi.org/10.1016/j. mineng.2009.10.002

Norgate TE, Rankin WJ (2000) Life cycle assessment of copper and nickel production. In: Mineprex 2000, 11-13 Sept., Melbourne. Australasian Institute of Mining and Metallurgy, Carlton, S $133-138$

Norilsk Nickel (2011) Metals in our lives. Annual review of MMC Norilsk Nickel for 2010. https://www.nornickel.com/upload/iblock/ de8/file1694.pdf. Zugegriffen: 1. Sept. 2020 (Hg. v. Norilsk Nickel. Moskau)

Norilsk Nickel (2014) Rethinking our strategic priorities. Annual report 2013. https://www.nornickel.com/upload/iblock/04e/nn_ar_ 2013_en_fin_na_20_iyunya.pdf. Zugegriffen: 1. Sept. 2020 (Hg. v. Norilsk Nickel. Moskau)

Northey S, Mohr S, Mudd GM, Weng Z, Giurco D (2014) Modelling future copper ore grade decline based on a detailed assessment of copper resources and mining. Resour Conserv Recycl 83:190-201. https://doi.org/10.1016/j.resconrec.2013.10.005

Northey SA (2018) Daten zum Energieverbrauch von Kupferminen, März 2018 (Email an N. Rötzer)

Northey SA, Haque N, Mudd GM (2013) Using sustainability reporting to assess the environmental footprint of copper mining.
J Clean Prod 40:118-128. https://doi.org/10.1016/j.jclepro.2012. 09.027

Rötzer N, Schmidt M (2018) Decreasing Metal Ore Grades-Is the Fear of Resource Depletion Justified? Resources 7(4):88. https:// doi.org/10.3390/resources 7040088

Rötzer N, Schmidt M (2020) Historical, current, and future energy demand from global copper production and its impact on climate change. Resources 9(4):44. https://doi.org/10.3390/ resources 9040044

Schäfer P (2021) Recycling - ein Mittel zu welchem Zweck? Modellbasierte Ermittlung der energetischen Aufwände des Metallrecyclings für einen empirischen Vergleich mit der Primärgewinnung. Springer, Wiesbaden

Schäfer P, Schmidt M (2020) Discrete-Point Analysis of the Energy Demand of Primary versus Secondary Metal Production. Environmental Science \& Technology 54(1):507-516. https://doi.org/ 10.1021/acs.est.9b05101

Schlesinger ME, King MJ, Sole KC, Davenport WG (2011) Extractive metallurgy of copper. Elsevier, Amsterdam

SEA-DISTANCES (2020) Sea distances-online tool for calculation distances between sea ports. https://sea-distances.org/. Zugegriffen: 15 . Sept. 2020

Vale (var.) Annual report. Jahre 2005-2016. VALE, Rio de Janeiro

Valero A (2008) Exergy evolution of the mineral capital on earth. Dissertation an der Universität Zaragoza

Vieira MDM, Goedkoop MJ, Storm P, Huijbregts MAJ (2012) Ore Grade Decrease As Life Cycle Impact Indicator for Metal Scarcity: The Case of Copper. Environ Sci Technol 46(23):12772-12778. https://doi.org/10.1021/es302721t

Wellmer F-W, Dalheimer W, Wagner M (2008) Economic evaluations in exploration, 2. Aufl. Springer, Berlin 